\title{
The fraction of first- and second-generation stars in globular clusters
}

\section{The case of NGC $6752^{\star}$}

\author{
E. Carretta
}

\author{
INAF - Osservatorio Astronomico di Bologna, via Ranzani 1, 40127 Bologna, Italy \\ e-mail: eugenio.carretta@oabo.inaf.it
}

Received 19 June 2013 / Accepted 16 July 2013

\begin{abstract}
We present a new method of estimating the percentage of stars with the chemical composition of first and second generations currently hosted in Galactic globular clusters (GCs). We compared cluster and field stars of similar metallicity in the $[\mathrm{Fe} / \mathrm{H}]-[\mathrm{Na} / \mathrm{H}]$ plane. Since the phenomenon of multiple populations is only restricted to the cluster environment, the number of GC stars whose location coincides with that of field stars provides the percentage of first-generation stars in that cluster. By exclusion, the number of secondgeneration stars is derived. We assembled a dataset of 1891 field stars of the thin disk, thick disk, and halo of the Milky Way in the metallicity range $-3.15 \leq[\mathrm{Fe} / \mathrm{H}] \leq+0.48$ dex and with $\mathrm{Na}$ abundance from high resolution spectra. They are mostly dwarfs, but include giants. Considering only the range in metallicity spanned by most GCs extensively studied for the Na-O anticorrelation $(-2.36 \leq[\mathrm{Fe} / \mathrm{H}] \leq-0.33$ dex $)$, we have 804 stars. The total sample is homogeneized by offsets in $[\mathrm{Fe} / \mathrm{H}]$ and $[\mathrm{Na} / \mathrm{H}]$ with respect to a reference sample using the same line list and non-local thermodynamic equilibrum (NLTE) correction for Na adopted in a recent extensive survey of GC stars. This fully accounts for offsets among analyses due to different temperature scales, line lists, adopted (or neglected) corrections for departures from LTE. We illustrate our method estimating the number of first- and second-generation stars in the well studied GC NGC 6752. As a by-product, the comparison of $[\mathrm{Na} / \mathrm{H}]$ values in $\mathrm{GC}$ and field stars suggests that at least two classes of old stellar systems probably contributed to the halo assembly: one group with characteristics similar to the currently existing GCs, and the other more like the present-day dwarf satellite galaxies.
\end{abstract}

Key words. stars: abundances - stars: Population II - Galaxy: abundances - Galaxy: stellar content - globular clusters: general globular clusters: individual: NGC 6752

\section{Introduction}

Once upon a time, the Galactic globular clusters (GCs) were considered the best example in nature of simple stellar populations (SSP, see e.g. the review by Renzini and Fusi Pecci 1988). The pioneering studies by Cohen (1978) and Peterson (1980), however, showed that supposedly coeval stars in GCs do not have the same initial chemical composition, which is a prerequisite, by definition, of SSPs. In particular, Cohen (1978) concluded that "the primordial gas in M3 was not chemically homogeneous during the time interval when the star formation of the currently observed M3 red giants took place."

In both studies a scatter of $\mathrm{Na}$ abundances in cluster stars was noted, and $\mathrm{Na}$ is still one of the best tracers used to characterize the peculiar chemistry of stars in GCs. Different amounts of $\mathrm{Na}$ excess, anticorrelated with varying degrees of $\mathrm{O}$ depletion, were found in all GCs studied by the Lick-Texas group (see Kraft 1994 for a review). The discovery of the Na-O anticorrelation, coupled to the C-N anticorrelation already widely studied among cluster giants (e.g. Smith 1987), led to identifying the nucleosynthesis source responsible for the observed changes in the network of proton-capture reactions in H-burning at high temperatures. The NeNa cycle was expected to operate, producing $\mathrm{Na}$, in the same region where the ON part of the $\mathrm{CNO}$ cycle

* Table 2 is only available at the CDS via anonymous ftp to cdsarc.u-strasbg. fr (130.79.128.5) or via

http://cdsarc.u-strasbg.fr/viz-bin/qcat?]/A+A/557/A128 is fully active (Denisenkov \& Denisenkova 1989; Langer et al. 1993), thereby destroying O. The abundance variations were found to only be restricted to the dense environment of GCs, since Gratton et al. (2000) demonstrated that Na, O abundances are not modified in field stars from the typical levels established by supernovae ( $\mathrm{SNe}$ ) nucleosynthesis only.

However, at the time it was unclear where the hot H-burning was located, whether in the stars currently observed in GCs or outside. The finding of a Na-O anticorrelation (as well as starto-star variations in $\mathrm{Mg}$ anticorrelated to those in $\mathrm{Al}$ ) among unevolved stars in NGC 6752 (Gratton et al. 2001) unambigously answered this question. These scarcely evolved stars do not reach high enough temperatures to efficiently produce $\mathrm{Na}$ or $\mathrm{Al}$ in their interiors; moreover, their convective envelope only covers a negligible fraction of their mass. The milestone established by Gratton et al. (2001) was to show that the chemical pattern observed in dwarfs currently in GCs must have been necessarily imprinted in the gas from which they formed by the most massive stars of a previous stellar generation. Observing Na-O variations in cluster stars means that we are in the presence of multiple stellar generations, no more, no less.

The new paradigm for GCs as examples of multiple stellar populations has already been assessed well, and much work has been done in recent years (see the exhaustive reviews by Gratton et al. 2004, 2012): chemical tagging of different generations, identification of multiple photometric sequences in the colour-magnitude diagrams (CMDs), and determination of the impact of modified chemical composition on photometric bands 
(e.g., Carretta et al. 2011; Sbordone et al. 2011; Milone et al. 2012; Cassisi et al. 2013). However, much still remains to be done, in particular to better quantify the properties of different stellar generations, such as the ratio between the fraction of first and second generation stars that currently compose the cluster populations. This ratio is a fundamental parameter in several key issues related to the formation of GCs: their dynamical evolution and spatial mixing (Vesperini et al. 2013) and their connection to the formation of the Galactic halo (Carretta et al. 2010; Vesperini et al. 2010; Martell et al. 2011).

The first quantitative estimate was possible thanks to the extensive FLAMES survey of GCs (Carretta et al. 2006, 2009a,b, and ongoing follow up). The homogeneous dataset of $\mathrm{Na}, \mathrm{O}$ abundances for more than 1500 giant stars in about 20 GCs allowed Carretta et al. (2009a) to show that only one third of the stars currently observed in GCs have a chemistry of firstgeneration, primordial stars, and that the bulk $(\sim 70 \%)$ belongs to the second generation. Stars were assigned to the primordial (P) component if their $\mathrm{O}$ and $\mathrm{Na}$ content was similar to that of field stars with the same metallicity $[\mathrm{Fe} / \mathrm{H}]^{1}$. Stars deviating from the high-O, low-Na locus were considered secondgeneration stars of an intermediate (I) or extreme (E) component according to their $[\mathrm{O} / \mathrm{Na}]$ ratio.

Recently, Milone et al. (2013) have criticized the criteria used in Carretta et al. (2009a) as arbitrary, because in the case of NGC 6752 the three discrete groups identified in Carretta et al. $(2011,2012)$ did not match the subdivisions found from the abundances by Yong et al. (2003, 2005). However, the separation between first and second generation in Carretta et al. (2009a) is not arbitrary; the $\mathrm{P}$ component includes all stars with $[\mathrm{Na} / \mathrm{Fe}]$ ratios in the range between $[\mathrm{Na} / \mathrm{Fe}]_{\min }$ and $[\mathrm{Na} / \mathrm{Fe}]_{\min }+0.3$, where 0.3 dex corresponds to around four times the star-tostar error on $[\mathrm{Na} / \mathrm{Fe}]$ in each cluster, so that this group includes all stars with $\mathrm{Na}$ abundances similar to those of field stars ${ }^{2}$. The method used in Carretta et al. (2009a) may suffer from two possible shortcomings. First, it is only based on stars on the Na-O anticorrelation in each cluster, i.e., with measured abundances for both $\mathrm{O}$ and $\mathrm{Na}$, even if the separation first-/second-generation is made using only $\mathrm{Na}$ abundances. Second, the minimum ratio $[\mathrm{Na} / \mathrm{Fe}]_{\min }$ is estimated by eye, assuming that the stars with lowest $\mathrm{Na}$ abundances along the anticorrelation (possibly excluding a few outliers) have the typical composition of normal halo stars, with no direct comparison with field stars.

In the present study, therefore, we present a simple variation of that method, to overcome its possible uncertainties. We propose to compare the location of Galactic field stars in the $[\mathrm{Na} / \mathrm{H}]$ vs. $[\mathrm{Fe} / \mathrm{H}]$ plane, fixing the level from SNe nucleosynthesis alone, to the position of giants in GCs. The excess of $\mathrm{Na}$ will clearly select second-generation stars with modified composition. This approach does not rely directly on the Na-O anticorrelation, so it may make use of all stars with measured $\mathrm{Na}$ abundances in a GC, usually a larger number than those with $\mathrm{O}$ abundances, which are more difficult to measure $(\mathrm{O}$ is depleted, whereas $\mathrm{Na}$ is enhanced in proton-capture reactions).

In the present paper we present the assembling, over the metallicity range spanned by Galactic GCs, of the comparison

\footnotetext{
1 We adopt the usual spectroscopic notation, i.e. $[X]=\log (X)_{\text {star }}$ $\log (X)_{\odot}$ for any abundance quantity $X$, and $\log \epsilon(X)=\log \left(N_{\mathrm{X}} / N_{\mathrm{H}}\right)+$ 12.0 for absolute number density abundances.

2 The separation between the I and E fractions in second-generation stars, at $[\mathrm{O} / \mathrm{Na}]=-0.9$ dex, is somewhat arbitrary, but this value corresponds to the minimum or sudden drop in the distribution function of $[\mathrm{O} / \mathrm{Na}$ ] for GCs with a long tail of very O-poor stars (NGC 2808, NGC 3201, NGC 5904).
}

sample of field stars (Sect. 2), using several literature studies, which were all shifted on a system defined by our reference sample (Gratton et al. 2003). The outliers in the $[\mathrm{Na} / \mathrm{H}]-[\mathrm{Fe} / \mathrm{H}]$ distribution are discussed in Sect. 3, and the application of the present method is tested in Sect. 4 using NGC 6752, one of the closest and best studied GC, as a template. As a by-product of this comparison, some final considerations on the candidate building blocks that may have contributed to the formation of the Galactic halo are given in Sect. 4.

The abundances of $\mathrm{Na}$ in the reference sample by Gratton et al. (2003) are obtained with the same line lists, and include the same prescriptions for NLTE corrections to Na values, used in the FLAMES survey of GCs which at present is the largest survey of homogeneous abundances in GC giants. Already $21 \mathrm{GCs}$ (soon to become 24 with the addition of NGC 362, NGC 4833, and NGC 6093) are analysed and this offers a unique opportunity. In a forthcoming paper we will apply the method developed here to derive homogeneous estimates of the fraction of first- and second-generation stars in all the GCs of this sample, to compare them with previously derived values from spectroscopy and/or photometry, and to study their relationship with global cluster properties.

\section{The comparison sample of field stars}

To assemble the comparison sample of field stars we searched the literature for the most recent studies based on high resolution spectroscopy that provide $\mathrm{Na}$ abundances for a large number of stars with different metallicities. Our method of estimating the fraction of stars of different generations in GCs is based on comparing the location of cluster and field stars in the $\mathrm{Fe}-\mathrm{Na}$ plane; as a result, the main requisites are homogeneity of measurements and a good sampling in this plane to highlight possible outliers. In particular, our aim was to secure good coverage in $[\mathrm{Fe} / \mathrm{H}]$ over the range in metallicity spanned by the bulk of GCs.

\subsection{Our approach: problems and methods}

About twenty abundance analyses with these characteristics could be easily picked up in the past 15 years, with a number of stars analysed that range from 23 metal-poor giants in Johnson (2002) to more than 1000 FGK dwarfs surveyed with HARPS in Adibekyan et al. (2012).

Obviously, independent studies designed to study different stellar populations and to deal with different astrophysical problems involve a series of different methods and assumptions that in turn may affect the requirement of homogeneity. Among these we may include different temperature scales, based on photometry or derived from the spectra, and differences in the adopted reference solar abundances or on the scales of atomic parameters. The latter is less important for the $\mathrm{Na}$ I lines most used in abundance analyses, since their $g f$ s are very well known and homogeneously used, but may represent a source of discrepancy concerning $\mathrm{Fe}$, especially when coupled to different temperature scales. Another potentially dangerous source of offset among different studies are the corrections for departures from the LTE assumptions, in particular for Na. This problem may become relevant, especially when abundances derived for warm dwarfs are compared to those obtained in metal-poor, cool stars of low gravity, where the NLTE effects are stronger (see e.g. Baumüller et al. 1998; Gratton et al. 1999; Korotin \& Mishenina 1999; Mashonkina et al. 2000; Takeda et al. 2003; Gheren et al. 2004; Andrievsky et al. 2007; Lind et al. 2011, and references 


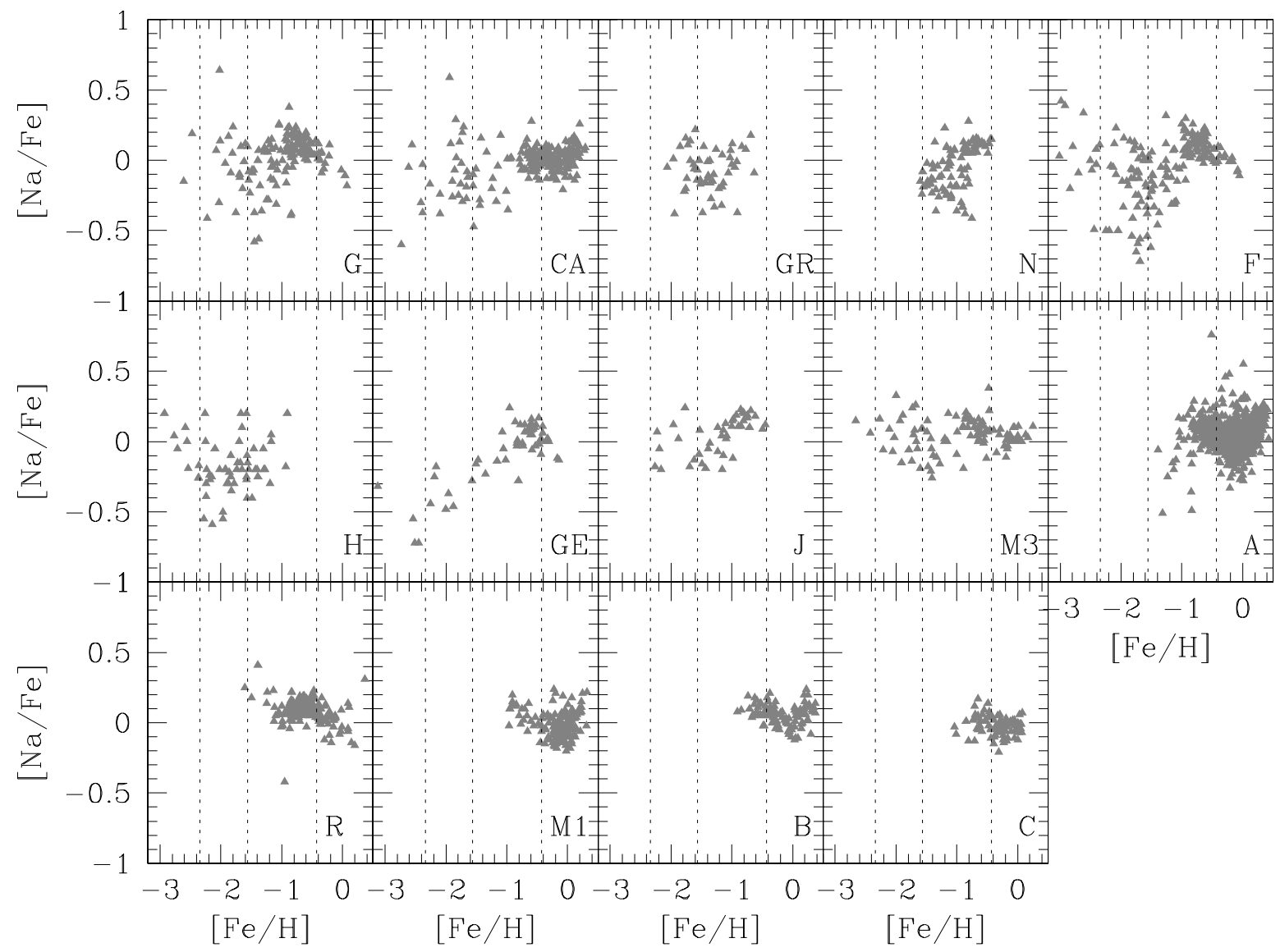

Fig. 1. $[\mathrm{Na} / \mathrm{Fe}]$ abundance ratios as a function of $[\mathrm{Fe} / \mathrm{H}]$ for the 14 samples selected from the literature. The code-labels are listed in Table 1 . The $[\mathrm{Na} / \mathrm{Fe}]$ and $[\mathrm{Fe} / \mathrm{H}]$ values are those of the original papers. The leftmost and rightmost dotted lines indicate the metallicity range of the bulk of GCs in the FLAMES survey (see e.g. Carretta et al. 2009a,b). The line at $[\mathrm{Fe} / \mathrm{H}]=-1.56$ dex shows the metallicity of NGC 6752 , our test case.

therein). Moreover, different groups adopt different prescriptions for NLTE corrections.

Previous extensive compilations of literature data by Venn et al. (2004; 821 stars) and by Soubiran \& Girard (2005; 743 stars) were designed to deal with specific problems (mainly related to the study of Galactic stellar populations) and are not very suitable to our purposes for various reasons. Venn et al. did not try to homogenise the abundance data apart from atomic data for a few neutron-capture elements (Y, Ba, and Eu). Moreover, in their sample they did not include the study by Gratton et al. (2003), which we adopt as our reference sample (see below, Sect. 2.2). Soubiran and Girard used stars in common among their 11 selected samples to operate an homogeneisation of the total sample. However, their study was focussed on the interface between thick and thin disks, so they restricted the metallicity range to stars more metal rich than $[\mathrm{Fe} / \mathrm{H}]=-1.3$ dex. They also intended to avoid the larger scatter observed at low metallicities. Moreover, to further reduce the observed dispersion, they limited their sample to stars hotter than $4500 \mathrm{~K}$. However, one of our aims is to also provide a good sampling in the low metalabundance regime; since our main purpose is to build a sample to be compared mainly to giant stars in rather metal-poor objects such as the GCs, the quoted existing compilations are unsuitable (apart from the missing recent studies performed after 2005).

From our literature search, we eventually selected 14 studies, according to criteria defined below in Sect. 2.4. In Fig. 1 we show the ratio $[\mathrm{Na} / \mathrm{Fe}]$ as a function of the metallicity $[\mathrm{Fe} / \mathrm{H}]$ for all these samples, using the original values in each paper.
In Table 1 we listed a one/two letter coding for each sample, together with a summary of a few properties of the samples. The original studies cover all the major Galactic stellar populations, thin and thick disks, and halo. Most objects are dwarf stars, but also subgiants and giants are represented in some samples. In Col. 7 we indicated whether the abundance analysis for $\mathrm{Na}$ was made under the LTE assumption, or corrections for NLTE were applied. References in the latter case can be found in the original papers.

Aided by the reference lines at different metallicities, from Fig. 1 we first note that different samples occupy more or less the same position, indicating that offsets among different analyses are not severe. Second, there is some evidence of increased scatter in $[\mathrm{Na} / \mathrm{Fe}]$ ratios at low metallicity, in particular between $[\mathrm{Fe} / \mathrm{H}] \sim-2.3 \mathrm{dex}$ and $[\mathrm{Fe} / \mathrm{H}] \simeq-1.5 \mathrm{dex}$. This occurrence, already noted in several studies, can be appreciated particularly well in a few samples (Gratton et al. 2003; Fulbright 2000; and Hanson et al. 1998).

In the following analysis, however, we chose to switch to the plane $[\mathrm{Na} / \mathrm{H}]$ vs. $[\mathrm{Fe} / \mathrm{H}]$ for two main reasons. The first was simply to decouple $\mathrm{Na}$ and $\mathrm{Fe}$, avoiding that errors in Fe reflect on both axes. The second is to fully exploit the different sites of nucleosynthesis for $\mathrm{Na}$. The main production of $\mathrm{Na}$ is considered to be hydrostatic carbon burning in massive stars, a primary mechanism (see e.g. Woosley \& Weaver 1995). Since Na is an odd- $Z$ element, its production is a strong function of the neutron density, which is a function of the initial stellar metallicity (Truran \& Arnett 1971; Woosley \& Weaver 1995). A simple linear relation between $[\mathrm{Na} / \mathrm{H}]$ and $[\mathrm{Fe} / \mathrm{H}]$ is then expected. 
Table 1. Mean properties of the samples.

\begin{tabular}{|c|c|c|c|c|c|c|c|c|c|c|c|c|c|}
\hline & $\begin{array}{l}\text { Nr. } \\
\text { stars }\end{array}$ & $\begin{array}{l}\text { With } \\
\mathrm{Na}\end{array}$ & $\begin{array}{c}\text { Min. } \\
{[\mathrm{Fe} / \mathrm{H}]}\end{array}$ & $\begin{array}{c}\text { Max. } \\
{[\mathrm{Fe} / \mathrm{H}]}\end{array}$ & $\begin{array}{l}\text { Evol. } \\
\text { stage }\end{array}$ & $\begin{array}{l}\text { LTE or } \\
\text { NLTE }\end{array}$ & Pop.1 & Pop. 2 & Pop.3 & $\begin{array}{c}\text { Comm. } \\
\text { stars }\end{array}$ & $\begin{array}{c}\text { Mean off. } \\
{[\mathrm{Fe} / \mathrm{H}]}\end{array}$ & $\begin{array}{c}\text { Mean off. } \\
{[\mathrm{Na} / \mathrm{H}]}\end{array}$ & Ref. \\
\hline G & 150 & 147 & -2.61 & +0.07 & $\begin{array}{c}\text { dwarfs, } \\
\text { SGB }\end{array}$ & NLTE & $\begin{array}{l}\text { accret. } \\
\text { comp. }\end{array}$ & $\begin{array}{l}\text { dissip. } \\
\text { comp. }\end{array}$ & $\begin{array}{l}\text { thin } \\
\text { disk }\end{array}$ & & & & Gratton et al. (2003) \\
\hline $\mathrm{CA}$ & 286 & 237 & -2.84 & +0.29 & $\begin{array}{c}\text { dwarfs, } \\
\text { giants }\end{array}$ & NLTE & halo & $\begin{array}{l}\text { thick } \\
\text { disk }\end{array}$ & $\begin{array}{l}\text { thin } \\
\text { disk }\end{array}$ & 41 & $\begin{array}{c}-0.10 \text { dex } \\
\sigma=0.07\end{array}$ & $\begin{array}{c}+0.01 \mathrm{dex} \\
\sigma=0.08\end{array}$ & Carretta et al. (2000) \\
\hline GR & 58 & 49 & -2.31 & -0.63 & $\begin{array}{c}\text { dwarfs, } \\
\text { giants }\end{array}$ & NLTE & halo & $\begin{array}{l}\text { thick } \\
\text { disk }\end{array}$ & & 14 & $\begin{array}{c}-0.13 \mathrm{dex} \\
\sigma=0.05\end{array}$ & $\begin{array}{c}-0.02 \mathrm{dex} \\
\sigma=0.09\end{array}$ & Gratton et al. (2000) \\
\hline $\mathrm{N}$ & 94 & 94 & -1.60 & -0.63 & dwarfs & LTE & halo & $\begin{array}{l}\text { thick } \\
\text { disk }\end{array}$ & & 35 & $\begin{array}{c}-0.05 \mathrm{dex} \\
\sigma=0.07\end{array}$ & $\begin{array}{c}-0.06 \mathrm{dex} \\
\sigma=0.04\end{array}$ & Nissen \& Schuster (2010) \\
\hline $\mathrm{F}$ & 178 & 174 & -3.01 & -0.05 & $\begin{array}{c}\text { dwarfs, } \\
\text { giants }\end{array}$ & LTE & halo & $\begin{array}{l}\text { thick } \\
\text { disk }\end{array}$ & $\begin{array}{l}\text { thin } \\
\text { disk }\end{array}$ & 110 & $\begin{array}{c}+0.05 \mathrm{dex} \\
\sigma=0.06\end{array}$ & $\begin{array}{c}+0.06 \mathrm{dex} \\
\sigma=0.05\end{array}$ & Fulbright (2000) \\
\hline $\mathrm{H}$ & 59 & 59 & -2.93 & -0.91 & giants & LTE & halo & & & 32 & $\begin{array}{c}+0.05 \mathrm{dex} \\
\sigma=0.12\end{array}$ & $\begin{array}{c}+0.27 \mathrm{dex} \\
\sigma=0.16\end{array}$ & Hanson et al. (1998) \\
\hline GE & 55 & 55 & -3.12 & -0.14 & dwarfs & NLTE & halo & $\begin{array}{l}\text { thick } \\
\text { disk }\end{array}$ & $\begin{array}{l}\text { thin } \\
\text { disk }\end{array}$ & 23 & $\begin{array}{c}-0.03 \mathrm{dex} \\
\sigma=0.05\end{array}$ & $\begin{array}{c}-0.00 \text { dex } \\
\sigma=0.07\end{array}$ & Gehren et al. (2006) \\
\hline $\mathrm{J}$ & 43 & 42 & -2.99 & -0.45 & $\begin{array}{l}\text { turnoff, } \\
\text { SGB }\end{array}$ & LTE & halo & $\begin{array}{l}\text { thick } \\
\text { disk }\end{array}$ & & 18 & $\begin{array}{c}+0.00 \mathrm{dex} \\
\sigma=0.08\end{array}$ & $\begin{array}{c}-0.03 \mathrm{dex} \\
\sigma=0.06\end{array}$ & Jonsell et al. (2005) \\
\hline M3 & 100 & 100 & -2.66 & +0.25 & $\begin{array}{c}\text { dwarfs, } \\
\text { giants }\end{array}$ & NLTE & halo & $\begin{array}{l}\text { thick } \\
\text { disk }\end{array}$ & $\begin{array}{l}\text { thin } \\
\text { disk }\end{array}$ & 34 & $\begin{array}{c}-0.04 \text { dex } \\
\sigma=0.09\end{array}$ & $\begin{array}{c}-0.11 \text { dex } \\
\sigma=0.17\end{array}$ & Mishenina et al. (2003) \\
\hline A & 1111 & 1110 & -1.39 & +0.55 & dwarfs & LTE & halo & $\begin{array}{l}\text { thick } \\
\text { disk }\end{array}$ & $\begin{array}{l}\text { thin } \\
\text { disk }\end{array}$ & 24 & $\begin{array}{c}-0.07 \mathrm{dex} \\
\sigma=0.07\end{array}$ & $\begin{array}{c}-0.08 \text { dex } \\
\sigma=0.11\end{array}$ & Adibekyan et al. (2012) \\
\hline $\mathrm{R}$ & 176 & 164 & -1.98 & +0.37 & dwarfs & LTE & halo & $\begin{array}{l}\text { thick } \\
\text { disk }\end{array}$ & $\begin{array}{l}\text { thin } \\
\text { disk }\end{array}$ & 43 & $\begin{array}{c}-0.07 \mathrm{dex} \\
\sigma=0.08\end{array}$ & $\begin{array}{c}-0.05 \mathrm{dex} \\
\sigma=0.08\end{array}$ & Reddy et al. (2006) \\
\hline M1 & 142 & 142 & -0.96 & +0.32 & dwarfs & LTE & $\begin{array}{l}\text { thick } \\
\text { disk }\end{array}$ & $\begin{array}{l}\text { thin } \\
\text { disk }\end{array}$ & $\begin{array}{c}\text { Her mov. } \\
\text { group }\end{array}$ & 10 & $\begin{array}{c}+0.01 \mathrm{dex} \\
\sigma=0.07\end{array}$ & $\begin{array}{c}+0.04 \mathrm{dex} \\
\sigma=0.06\end{array}$ & Mishenina et al. (2011) \\
\hline B & 102 & 102 & -0.91 & +0.37 & dwarfs & LTE & $\begin{array}{l}\text { thick } \\
\text { disk }\end{array}$ & $\begin{array}{l}\text { thin } \\
\text { disk }\end{array}$ & & 8 & $\begin{array}{c}-0.09 \mathrm{dex} \\
\sigma=0.14\end{array}$ & $\begin{array}{c}-0.05 \mathrm{dex} \\
\sigma=0.07\end{array}$ & Bensby et al. (2005) \\
\hline $\mathrm{C}$ & 90 & 81 & -1.04 & +0.08 & dwarfs & LTE & $\begin{array}{l}\text { thin } \\
\text { disk }\end{array}$ & & & 5 & $\begin{array}{c}+0.01 \mathrm{dex} \\
\sigma=0.04\end{array}$ & $\begin{array}{c}+0.06 \mathrm{dex} \\
\sigma=0.05\end{array}$ & Chen et al. (2000) \\
\hline
\end{tabular}

However, proton captures on ${ }^{22} \mathrm{Ne}$ in $\mathrm{H}$-burning at a high temperature result in the synthesis of $\mathrm{Na}$, which is able to explain the excess of Na observed in GC stars (Denisenkov \& Denisenkova 1989). Moreover, Langer et al. (1993) proposed that synthesis of ${ }^{23} \mathrm{Na}$ on the abundant isotope ${ }^{20} \mathrm{Ne}$ may strongly contribute to the Na production via proton capture. We can exploit these different production mechanisms for $\mathrm{Na}$ to our purposes.

Therefore, the $[\mathrm{Na} / \mathrm{H}]-[\mathrm{Fe} / \mathrm{H}]$ plane could be considered one of the best diagnostics for separate the stars with chemical signature from plain supernova nucleosynthesis (both in the Galactic field populations and in GCs) from stars where the proton capture reactions have acted to modify the original chemical imprinting. Any excess of $[\mathrm{Na} / \mathrm{H}]$ in $\mathrm{GC}$ stars, with respect to the linear behaviour in field stars at any given metallicity, can be interpreted as a signature of second-generation stars.

The run of the $[\mathrm{Na} / \mathrm{H}]$ ratios as a function of metallicity is shown in Fig. 2 for all the 14 datasets used to build our total comparison sample of field stars. Possible systematics among them owing to the different scales and methods adopted in the original studies were treated by means of offsets computed using stars in common to bring all the $[\mathrm{Na} / \mathrm{H}]$ and $[\mathrm{Fe} / \mathrm{H}]$ values onto a homogeneous system defined by a reference sample, and corroborated by an extended reference sample, as discussed below.

\subsection{The reference sample: Gratton et al. (2003)}

Our privileged reference sample is the dataset of 150 field subdwarfs and early subgiant stars with accurate parallaxes analysed by Gratton et al. (2003; hereinafter G, see Table 1 for details). That work was based on high resolution spectra for about 50 stars and on a collection of high quality equivalent widths (EWs) from the studies by Nissen \& Schuster (1997), Prochaska et al. (2000), and Fulbright (2000).

There are several reasons to choose this set as reference sample.

- Adopted solar reference abundances for $\mathrm{Na}$ (6.21) and Fe (7.54) are the same as used in the FLAMES survey of $\mathrm{Na}, \mathrm{O}$ abundances in GCs (including homogeneous abundances for almost 25 clusters).

- In the same vein, the atomic parameter scale and line list are the same for field stars in $\mathrm{G}$ and cluster stars; as said above, this is not very relevant for $\mathrm{Na}$, but it could be an advantage for the iron abundances.

- The grid for corrections from NLTE effects used for $\mathrm{Na}$ (from Gratton et al. 1999) is the same as adopted for $\mathrm{Na}$ abundances in GC stars of the above extensive survey.

- The G sample homogeneously covers all the metallicity range relevant for $\mathrm{GCs}$, going from $[\mathrm{Fe} / \mathrm{H}]=-2.61$ dex up to +0.07 dex.

Overall, considering the obvious differences due to dealing with nearby field stars rather than with distant cluster stars, the $\mathrm{G}$ sample looks like the best reference sample for to defining a common system for our total comparison sample. 


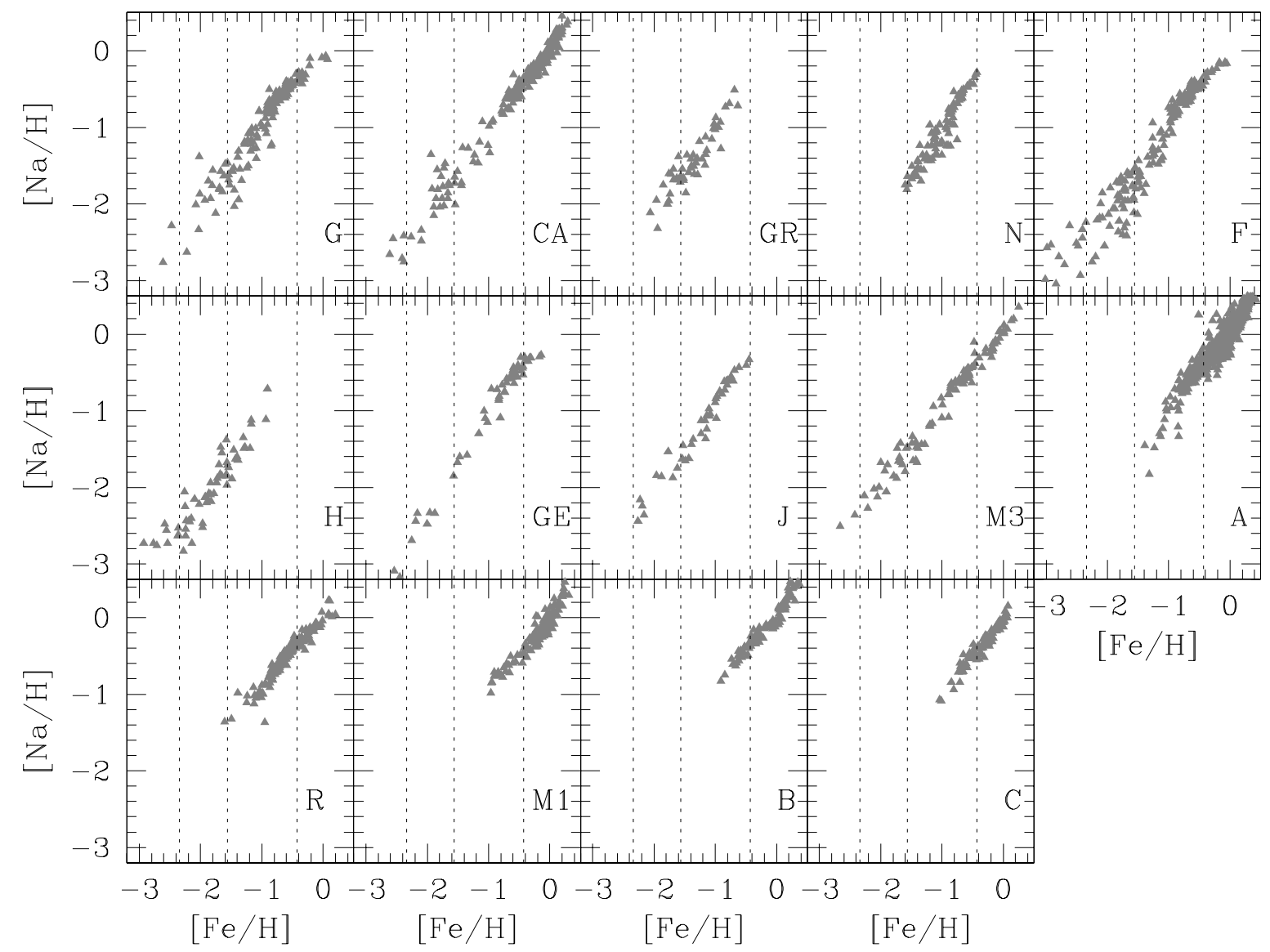

Fig. 2. As in Fig. 1 but for the $[\mathrm{Na} / \mathrm{H}]$ ratios as a function of $[\mathrm{Fe} / \mathrm{H}]$ for the 14 samples selected from the literature.

All the main stellar populations in the Galaxy are sampled in G: thin disk, thick disk, and halo. However, Gratton et al. (2003) did not adopt this classical classification. Using the orbital characteristics of the sample they distinguished stars belonging to a dissipative component (as proposed, e.g., by Eggen et al. 1962, and including objects from the classical populations of halo and thick disk) and stars of an accretion component, as in the scenario proposed by Searle \& Zinn (1978) for halo GCs. This classification scheme is being used more and more, thanks to large-scale surveys such as the SDSS, which stimulated many observational and theoretical works (see, e.g., Carollo et al. 2013 and Zolotov et al. 2009 for the dual halo nature). The relevance of the accretion versus dissipative-collapse component will be clear when discussing the outliers in the field star total sample (see Sect. 3 below).

A possible shortcoming of this reference sample is that it does not include giant stars, which are the vast majority of stars usually observed in distant GCs. We will see that this is not a source of concern because we can tie together the worlds of giants and dwarfs by using the sample of unevolved stars observed in GCs, in particular in our test case NGC 6752.

However, to ensure the reliability of the comparison sample of field stars, we defined an extended reference sample that (i) provides a larger number of stars for the cross-match with other studies for the homogeneisation of the different datasets, and (ii) includes a number of giants to check that in the $[\mathrm{Na} / \mathrm{H}]-[\mathrm{Fe} / \mathrm{H}]$ plane they occupy the same region of dwarfs, despite, say, the different amounts of correction for NLTE on $\mathrm{Na}$.

\subsection{The extended reference sample}

To build up our extended reference sample, we considered the study of about 300 field stars by Carretta et al. (2000; hereinafter code $\mathrm{CA}$ ), in the metallicity range from $[\mathrm{Fe} / \mathrm{H}] \simeq-2.8$ dex to $[\mathrm{Fe} / \mathrm{H}] \simeq+0.3 \mathrm{dex}$ (see Table 1 ) encompassing the metal abundance distribution of GCs. CA analysed or reanalysed stars using EW from high resolution spectra using homogeneous methods. The main advantages of adding this study are that both giants and dwarfs are included in the sample, the corrections for NLTE effects on $\mathrm{Na}$ are from the same grid in Gratton et al. (1999) used by $\mathrm{G}$, and more than 40 stars are in common with $\mathrm{G}$, allowing precise offsets be computed to compensate for the different scales of atmospheric parameters and linelists. Finally, the CA sample includes the reanalysis of the extensive set of disk stars by Edvardsson et al. (1993). This sample is present in both the large compilations by Venn et al. (2004) and Soubiran \& Girard (2005), but we preferred to use the dataset as reanalysed by CA to ease the homogeneisation of the final sample.

Adopting the CA sample we may check for possible systematic differences between dwarfs and giants, such as those due to the corrections for NLTE effects on Na. Owing to large ionising fluxes and high densities, the corrections are usually less important for unevolved stars than for giants.

In Fig. 3 (left panels) we show the temperature-gravity diagram for stars in the CA sample, divided into two subsamples of different metallicity at $[\mathrm{Fe} / \mathrm{H}]=-1$ dex. While the higher metallicity subsample only contains dwarfs (mostly based on the reanalysis of the Evdardsson et al. dataset), both giants and unevolved stars are present at low metallicity. The 


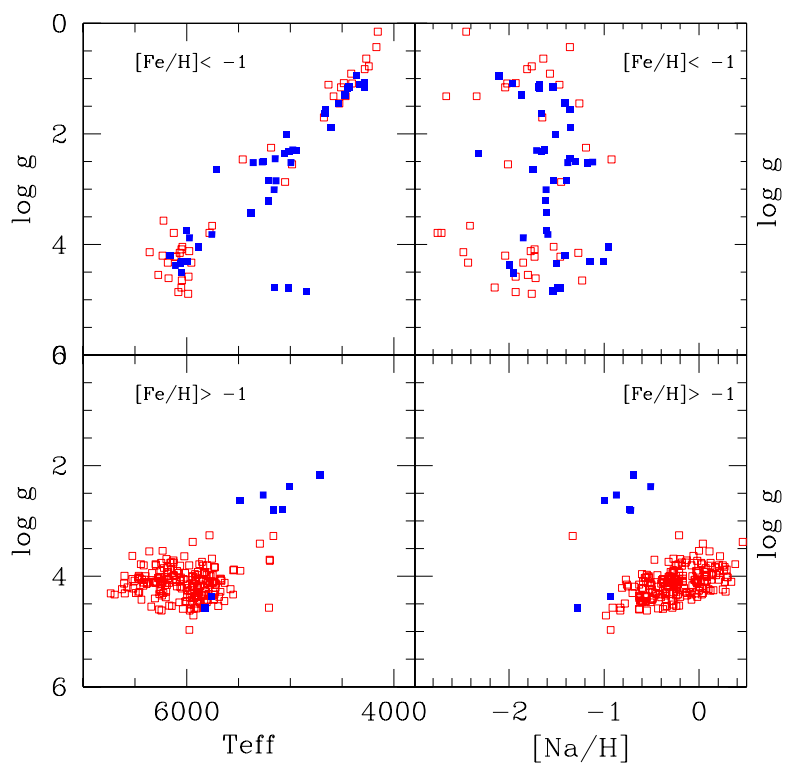

Fig. 3. Left panels: surface gravity as a function of effective temperature for stars in the CA sample (open squares) and in the GR sample (blue filled squares). In the upper panel only metal-poor stars $([\mathrm{Fe} / \mathrm{H}<-1 \mathrm{dex})$ are plotted, while in the lower panel stars have $[\mathrm{Fe} / \mathrm{H}]>-1$ dex. Right panels: the surface gravity is plotted as a function of $[\mathrm{Na} / \mathrm{H}]$, with the same symbols and metallicity ranges used in the left panels. Na abundances are corrected for departures from LTE according to Gratton et al. (1999) in both samples.

values of $[\mathrm{Na} / \mathrm{H}]$, including corrections for NLTE effects from Gratton et al. (1999), are plotted in the right-hand panels as a function of the gravity. No offset in $\mathrm{Na}$ abundances can be seen between low and high gravity stars.

To further reinforce our reference sample, we also added another study from the group of Gratton and collaborators, including in our extended reference sample the about 60 stars analysed by Gratton et al. (2000; hereinafter code GR, see Table 1 for further details). This is a set of stars mostly in the metallicity range $-2 \leq[\mathrm{Fe} / \mathrm{H}] \leq-1$ dex, with well determined evolutionary phases from the main sequence up to the upper red giant branch. Again, as in CA, the temperature scale and the linelist are different from those adopted by G, but the NLTE corrections applied to $\mathrm{Na}$ are from the same grid used by $\mathrm{G}$ and $\mathrm{Ca}$.

At low metallicity the sequence in the $T_{\text {eff }}-\log g$ plane is well defined (Fig. 3), and a good sampling of all the evolutionary phases is evident. No difference in the locus occupied by stars in the different phases is visible in the right-hand panels, even among the much more limited metal-rich subset.

Thus, we adopted the $\mathrm{G}$ sample as our favourite reference sample, which, in combination with the CA and GR datasets, constitutes our extended reference sample. These three sets are separated in Table 1 from the other samples.

\subsection{The final comparison sample}

From our literature search we only retained the studies with at least five analysed stars in common with our reference sample $G$ or more than ten objects in common with the total extended sample. Apart from the three already quoted samples, the studies we used were Hanson et al. (1998, hereinafter code H), Fulbright (2000; F), Chen et al. (2000; C), Mishenina et al. (2003; M3), Jonsell et al. (2005; J), Bensby et al. (2005; B), Gehren et al. (2006; GE), Reddy et al. (2006; R), Nissen \& Schuster (2010; N),
Mishenina et al. (2011; M1), Adibekyan et al. (2012; A). The code labels, the total number of stars and those with $\mathrm{Na}$ abundances, the metallicity range, the main evolutionary stage, whether the Na abundance analysis was or was not in LTE, and the main Galactic populations sampled in each study are indicated in the first ten columns of Table 1. In Col. 11 the number of stars in common between each original study and the reference sample $\mathrm{G}$ is listed.

By using these stars in common we computed the offsets in $[\mathrm{Na} / \mathrm{H}]$ and $[\mathrm{Fe} / \mathrm{H}]$, applying, when required, a $2.5 \sigma$-clipping with respect to the mean offset. The resulting average values (in the sense: $\mathrm{G}$ minus other sample) are listed in Cols. 12 and 13, together with the rms scatter about the mean. The amount of these offsets is generally not very large and scarcely significant, given the associated scatters. An exception is the offset in Na measured for the $\mathrm{H}$ sample, 0.27 dex (from 25 stars after $\mathrm{k} \sigma$-clipping). $\mathrm{Na}$ abundances in $\mathrm{H}$ are actually those obtained by Pilachowski et al. (1996); by using their sensitivity Table 7, if the discrepancy was due to uncorrect values of temperature and gravity, the required differences would be $386 \mathrm{~K}$ in $T_{\text {eff }}$ or 2.7 dex in $\log g$, while the observed mean offset are only $-24 \mathrm{~K}(\mathrm{rms}=105 \mathrm{~K})$ and $-0.06 \mathrm{dex}(\mathrm{rms}=0.42 \mathrm{dex})$, respectively. We thus cannot offer an explanation at present, but only note that a wide scatter in $\mathrm{Na}$ abundances has already been claimed by Pilachowski et al. (1996). Moreover, H is the only sample where no stars in common with G exist, but only 21 stars in common with CA and 11 with GR are available. However, the application of the derived offsets brings the $\mathrm{H}$ measurements in better agreement with the other samples. Including the $\mathrm{H}$ sample is valuable for reasons discussed below.

In absolute value, the mean offsets in temperature and gravity for the 13 samples are $\left|T_{\text {eff }}\right|=49, \sigma=30 \mathrm{~K}$ and $|\log g|=$ $0.09, \sigma=0.06$ dex. Using the sensitivities of $\mathrm{Na}$ and $\mathrm{Fe}$ to variations in the atmospheric parameters listed in the original papers, we verified that the measured offsets with respect to the $\mathrm{G}$ scale translate into uncertainties not exceeding \pm 0.05 dex in $[\mathrm{Na} / \mathrm{H}]$ (and similar amounts in $[\mathrm{Fe} / \mathrm{H}]$ ).

The final sample was obtained by eliminating all objects without $\mathrm{Na}$ abundance and applying the proper offsets in $[\mathrm{Na} / \mathrm{H}]$ and $[\mathrm{Fe} / \mathrm{H}]$ to all stars in each dataset. Stars with multiple determinations were treated according to the following criteria: (i) all stars in the $G$ dataset were retained; (ii) for multiple measurements with no star from G, those in CA or GR samples were retained, if present, averaging their values if a star was measured in both samples; (iii) stars measured in more than one of the other samples were averaged.

The total final sample consists of 1891 individual, unique stars on the scale defined by the $G$ sample. Among these objects, 147 are from G, 200 from CA, 32 from GR, 39 from $\mathrm{N}, 30$ from $\mathrm{F}, 26$ from $\mathrm{H}, 14$ from GE, 9 from J, 21 from M3, 1014 from A, 88 from R, 80 from M1, 40 from B, 49 from $C$, and 102 are averaged values from different studies. The assembled dataset covers the metallicity range from $[\mathrm{Fe} / \mathrm{H}]=-3.15$ dex up to $[\mathrm{Fe} / \mathrm{H}]=+0.48 \mathrm{dex}$. When restricting the range to the interval $-2.36 \leq[\mathrm{Fe} / \mathrm{H}] \leq-0.33$ dex where the GCs studied in the FLAMES survey by Carretta et al. (2009a,b) are located, along with the bulk of GCs in general, the field star sample includes 804 stars. A large proportion of stars, especially at high metallicity, is from the sample A, devoted to the chemical analysis of dwarf stars from the HARPS GTO planet search programme. Objects outside the metallicity range most relevant for GCs could well be rejected, but we retained all the same in our final sample because it could be used in future to study the 


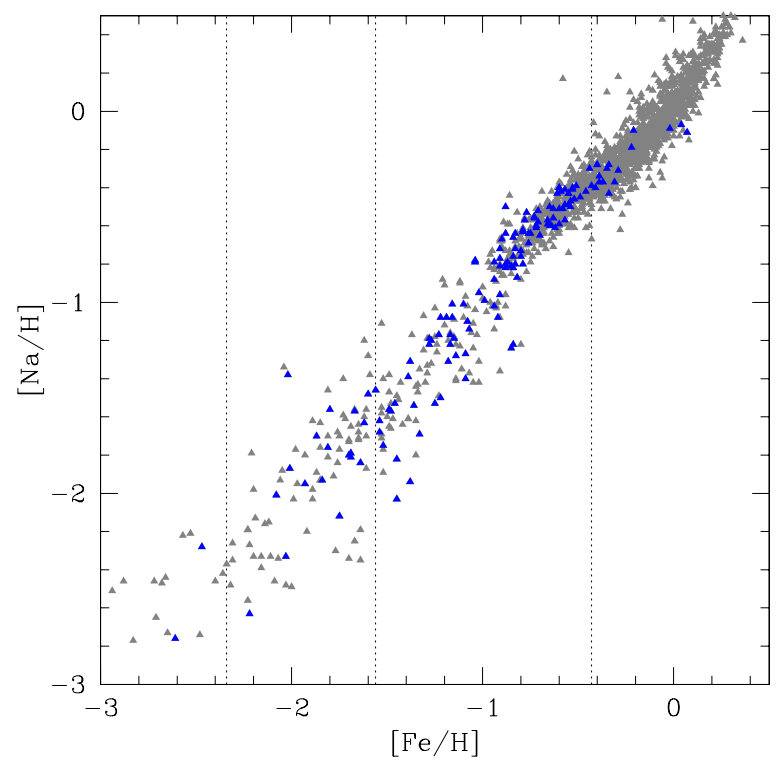

Fig. 4. $[\mathrm{Na} / \mathrm{H}]$ ratios as a function of the metallicity in our final sample, after correcting for the offsets in $\mathrm{Na}$ and $\mathrm{Fe}$ with respect to $\mathrm{G}$. Stars in the $\mathrm{G}$ sample are shown as blue symbols.

Galactic chemical evolution of $\mathrm{Na}$ using a large and homogeneous dataset.

In Table 2 we list an increasing order number, the star name, gravity, temperature, the $[\mathrm{Fe} / \mathrm{H}]$ and $[\mathrm{Na} / \mathrm{H}]$ values corrected to the $\mathrm{G}$ scale, the original sample code (AV means that the Fe and $\mathrm{Na}$ values from different samples were averaged), the Hipparcos name (when available; otherwise 000000 is listed), the right ascension and declination, referred to the 2000 equinox, as listed in SINBAD. This table will be available only in electronic form at the CDS database. Here we only present a few lines as an indication of its content.

The $[\mathrm{Na} / \mathrm{H}]$ values of this final sample are plotted as a function of metallicity in Fig. 4. Stars of the G sample are shown in this figure to show how the reference sample covers all the range in $[\mathrm{Fe} / \mathrm{H}]$ of interest for GCs well.

\section{Outliers in the field comparison sample}

There are stars in Fig. 4 that clearly deviate from the almost linear relation between $\mathrm{Na}$ and Fe. This occurrence has already been seen in Fig. 1 (as an increased scatter at low metallicity) and in Fig. 2 in the original samples, in particular in G, F, and $\mathrm{H}$. As a consequence, this behaviour cannot be a spurious effect of, say, the corrections applied to the original $[\mathrm{Na} / \mathrm{H}]$ and $[\mathrm{Fe} / \mathrm{H}]$ values to bring them onto the $\mathrm{G}$ system.

Since our method of deriving the number of secondgeneration stars is based on the excess of $\mathrm{Na}$ with respect to a baseline provided by field stars, it is important to better understand the nature of these outliers, especially because the deviation from linearity seems to become more and more relevant just at the metal abundance of our test case NGC 6752 $([\mathrm{Fe} / \mathrm{H}] \simeq-1.5)$.

We selected in Fig. 5 those stars that seem to deviate in $\mathrm{Na}$ at a given metallicity from the bulk of the distribution, to understand if there is some common feature that may explain the lower than average $\mathrm{Na}$ abundances. The selection of these outliers was made by eye, but is far from subjective and was checked by a linear fit in the $[\mathrm{Na} / \mathrm{H}]-[\mathrm{Fe} / \mathrm{H}]$ plane of all the 1891 stars in the final sample. The dispersion in $[\mathrm{Na} / \mathrm{H}]$ around this fit is $0.123 \mathrm{dex}$, and

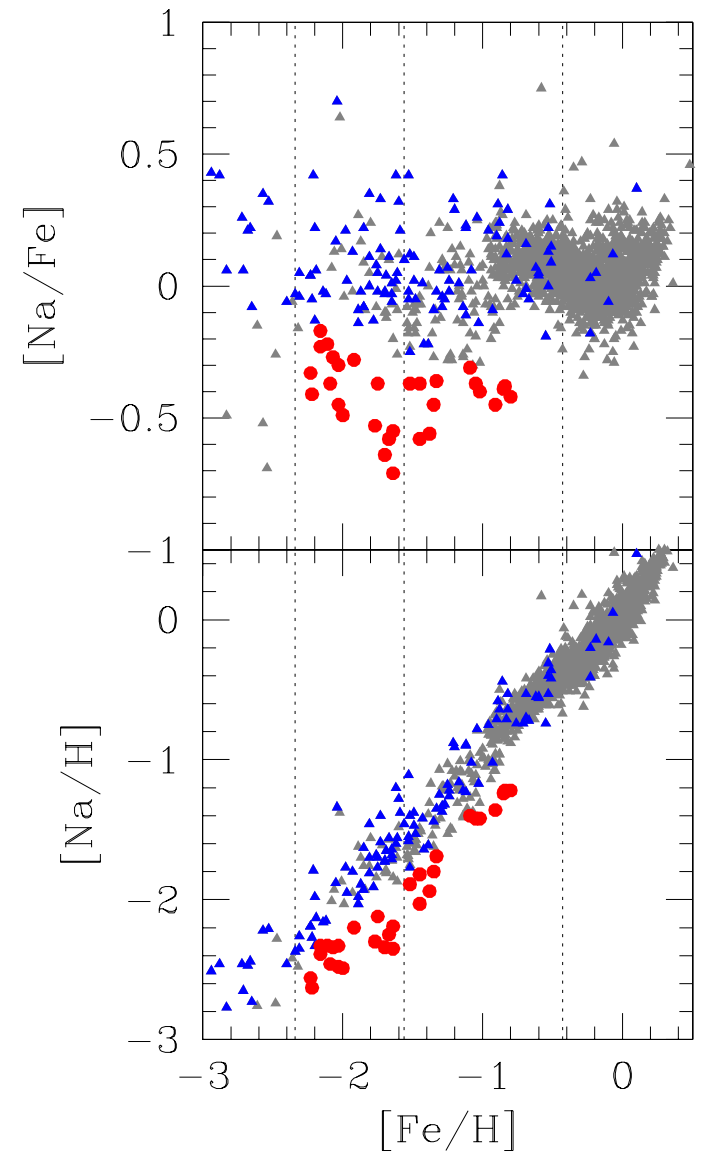

Fig. 5. Upper panel: $[\mathrm{Na} / \mathrm{Fe}]$ ratios as a function of metallicity for stars in the final homogenised sample (grey triangles for dwarfs and blue triangles for giants). Larger filled circles represent stars recognised as outliers with respect to the bulk of the distribution (with no distinction between dwarfs and the few giants). Dotted line have the same meaning as in previous figures. Bottom panel: the same, but for the ratios $[\mathrm{Na} / \mathrm{H}]$.

we checked that all candidate outliers have a sodium abundance from $\sim 2$ up to $5.8 \sigma$ lower than derived from the fit at any given metallicity ${ }^{3}$.

Among the 30 stars selected in Fig. 5, a large number (10 stars, $30 \%$ ) are from the $\mathrm{G}$ sample. The majority belong to the accretion component defined by $\mathrm{G}$ ( 8 stars out of 10$)$, with six found on retrograde orbits, as is the single star from GR included among the 30 selected stars. Six stars from N (including 3 objects in common with $\mathrm{G}$ and another star in common with A) are all of the low- $\alpha$ group defined in N (see also Schuster et al. 2012).

Another third of the stars in the selected set is from $\mathrm{F}$ (8 stars were also in common with $\mathrm{H}$, and 2 are average values with $\mathrm{H}$ and M3). Most of them (7 out of 10) are on retrograde orbits. Three other stars are unique objects from $\mathrm{H}$, and two of them were also found on retrograde orbits. All stars in this group from $\mathrm{F}$ are among the highest velocity stars in Fulbright (2002), who found a different patterm of abundances for them compared to lower velocity stars. In particular, the mean values for the $[\mathrm{Na} / \mathrm{Fe}]$ and $[\mathrm{Mg} / \mathrm{Fe}]$ were about 0.2 dex lower for the high velocity group (see Fig. 6 in Fulbright 2002).

3 The low dispersion around the best fit is driven by the large number of stars in the high metallicity regime. Using only stars more metal poor than $[\mathrm{Fe} / \mathrm{H}]=-0.3$ dex the r.m.s.scatter of the fit increases to $0.146 \mathrm{dex}$; in turn, the range of standard deviations spanned by the outliers with low Na decreases, but is, however, in the interval $1.3 \div 4.8 \sigma$. 
One of the three low- $\alpha$ stars studied by Ivans et al. (2003; BD+80 $245=$ HIP 40068) is included in this low-Na subset, as is the common proper motion pair HD 134439/40 whose formation was associated by King (1997) to the environment typical of dwarf spheroidals on the basis of the observed underabundances in $\alpha$-elements. All three stars have large apogalactic distances (about $20 \mathrm{kpc}$ for HIP 40068, Fulbright 2002; more than 40 for the pair HD 134439/40, King 1997).

It is clear that the sequence of stars selected with low $\mathrm{Na}$ abundances in Fig. 5 is predominantly composed of objects whose origin is attributed to accretion in the dichotomy of the Galactic halo amply studied by several groups (e.g. Hanson et al. 1998; Fulbright 2000, 2002; Stephens \& Boesgaard 2002; Gratton et al. 2003; Nissen \& Schuster 2010; Schuster et al. 2012, and references therein). It is convenient to adopt the terminology used by Nissen \& Schuster (1997) for these stars: they are better seen as iron-enriched than as $\alpha$ - or Na-poor. They formed in regions where the usual nucleosynthesis by type II SNe produced the known amount of $\alpha$-elements and $\mathrm{Na}$; however, the original fragments were probably small, not very dense and had a slow star formation rate. The low- $\alpha(\mathrm{Na})$ component therefore received chemical enrichment from both SNe II and SNeIa, owing to the slower chemical evolution timescales. The increased iron yields acted to dilute the overabundance of some products from the SNe II down to the observed level.

By contrast, the bulk of stars with higher $\mathrm{Na}$ (the high- $\alpha$ group in Nissen \& Schuster 1997, 2010, 2011; Schuster et al. 2012; the dissipative component in G) were generated from gas which experienced much more rapid chemical evolution, thus preserving the abundances of $\mathrm{Na}$ and $\alpha$-elements. This component may have been formed in more massive primordial fragments or participated in the early collapse of the proto-Galactic cloud. In the $[\mathrm{Na} / \mathrm{H}]-[\mathrm{Fe} / \mathrm{H}]$ plane, the latter component represents the bulk of our sample, $89 \%$ of stars in the metallicity range $-2.3<[\mathrm{Fe} / \mathrm{H}]<-0.8 \mathrm{dex}$ are Na-normal.

\section{Application of the method: the case of NGC 6752}

After setting the stage, it is time to introduce our main players, the multiple populations of GCs. We selected NGC 6752 to test the method. This is one of the closest GCs, often its stars are observed as calibrators in many spectroscopic studies, and it has been the target of several accurate abundance analyses and photometric studies as well. Among many other species, nitrogen abundances have been studied by Yong et al. (2008), whereas a detailed study of the $\mathrm{Mg}$ isotopes is found in Yong et al. (2003). Recently, three discrete stellar populations on the RGB were recognised first by Carretta et al. (2011) by using Grundahl's Strömgren photometry from Calamida et al. (2007); afterward, their different chemical composition was studied by Carretta et al. (2012). The discreteness of the photometric sequences was confirmed by Milone et al. (2013) with a larger set of photometric observations.

In the present context, we are interested in abundances of proton-capture elements, in particular $\mathrm{Na}$. One of the largest sample was analysed in Carretta et al. (2007), where Fe, Na, and $\mathrm{O}$ abundances were derived for about 150 giants using the multifibre FLAMES spectrograph. These data were complemented by Al, Mg, and Si abundances in Carretta et al. (2012). Smaller samples, but generally based on higher resolution and high signal-to-noise ratio $(\mathrm{S} / \mathrm{N})$ spectra, are from Yong et al. $(2005,2008)$ and Grundahl et al. (2002). We mainly employ the

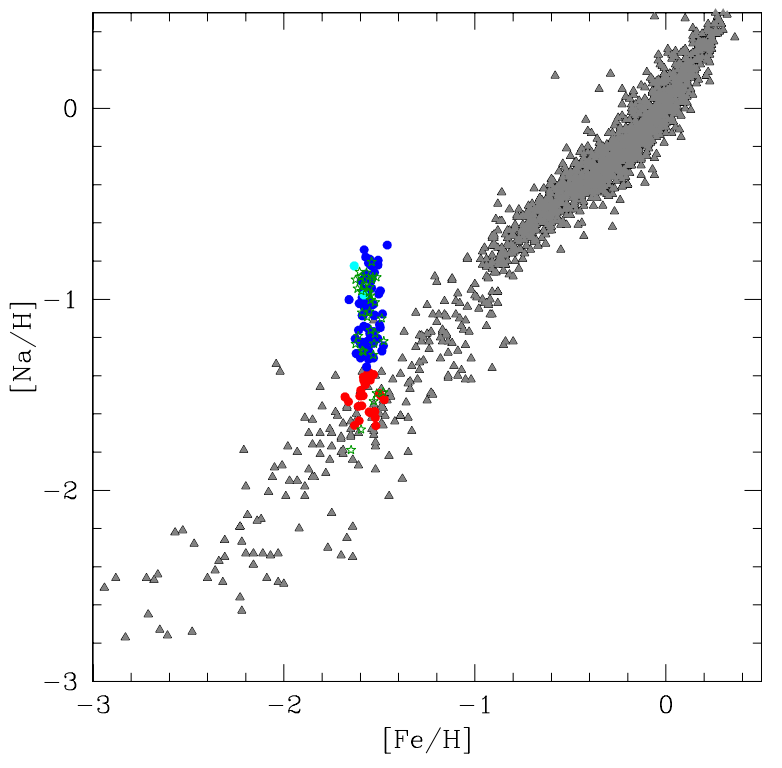

Fig. 6. Stars in NGC 6752 from Carretta et al. (2007) compared to our final sample (grey triangles). In NGC 6752 different symbols represent stars with measured $\mathrm{O}, \mathrm{Na}$ abundances of the primordial $\mathrm{P}$ component (red circles), the intermediate I component (blue circles), and the extreme E component (cyan circles) defined as in Carretta et al. (2009a). Green open star symbols indicate cluster stars with only $\mathrm{Na}$ abundance measured.

samples from Carretta et al. to better exploit the homogeneity of data.

The stars in NGC 6752 from Carretta et al. (2007) are compared to field stars in the present final sample in Fig. 6 to illustrate our method. Different symbols indicate the P, I, and E components as defined in Carretta et al. (2009a) by using stars with both $\mathrm{Na}$ and $\mathrm{O}$ abundances. Cluster stars with only Na measured are also indicated.

Even from this large scale figure it is evident that about two thirds of the stars in NGC 6752 clearly stand off the distribution of field stars, implying that these are actually stars with chemical compositions modified by processes involving protoncapture reactions and only restricted to the cluster environment.

The sample of cluster and field stars ultimately rest on the same scale concerning the line lists and NLTE corrections for $\mathrm{Na}$. However, the effective temperature scales adopted in Carretta et al. (2007) and in the reference sample $\mathrm{G}$ are different, and the presence of the low-Na field stars mainly from the accretion component may generate a doubt about the vertical normalization. To check this issue and nail down the samples we used stars with pure nucleosynthesis from $\mathrm{SNe}$, both in field and in NGC 6752, as follows.

We selected a range of \pm 0.2 dex in $[\mathrm{Fe} / \mathrm{H}]$ centred on the average metallicity of NGC $6752([\mathrm{Fe} / \mathrm{H}]=-1.56 \mathrm{dex}$, Carretta et al. 2009c). In Fig. 7 we then plotted as a function of the effective temperature the $[\mathrm{Na} / \mathrm{H}]$ ratios of all stars from Carretta et al. (2007) with $[\mathrm{Na} / \mathrm{Fe}]<+0.05 \mathrm{dex}$ and the four most Na-poor scarcely evolved stars from Carretta et al. (2005), three turn off stars and one subgiant ${ }^{4}$. This set of cluster stars was then chosen on purpose to represent the primordial stellar population in GCs, the one reflecting only nucleosynthesis from SNe.

4 These are stars studied in Gratton et al. (2001) and simply reanalysed in Carretta et al. (2005) by using updated line list and damping parameters, used afterward for GC giants. 
E. Carretta: Fraction of first and second generation stars

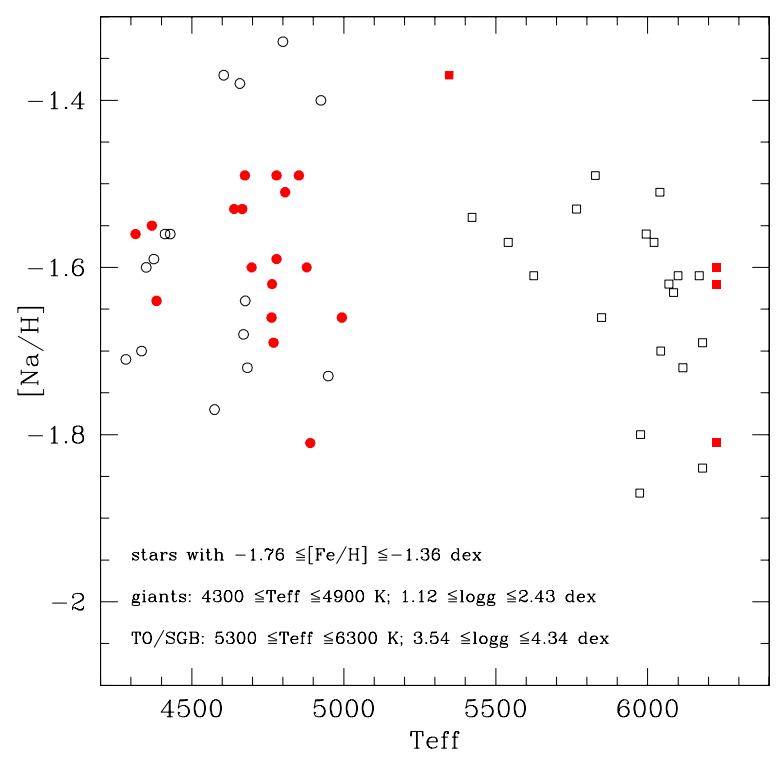

Fig. 7. $[\mathrm{Na} / \mathrm{H}]$ ratios as a function of effective temperature to test the concordance between field and cluster stars with nucleosynthesis from SNe. Filled symbols are stars in NGC 6752 from Carretta et al. (2005, 2007). Empty symbols are field stars in the present final sample. Circles indicate giants and squares represent dwarf/subgiant stars.

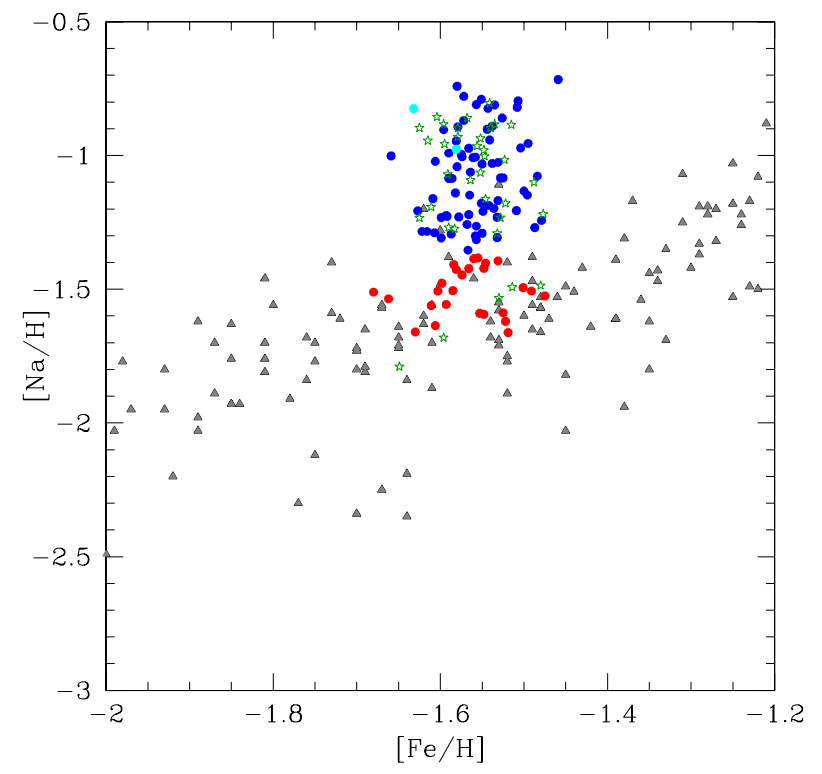

Fig. 8. Enlargement of Fig. 6 centred on the average metallicity of the globular cluster NGC 6752.

From the present final sample we then selected stars in the same metallicity range, and that match the temperature and gravity ranges of cluster stars. These field stars are plotted in Fig. 7, and they blend in with cluster stars very nicely. There is no systematic difference in the $[\mathrm{Na} / \mathrm{H}]$ content. Furthermore, the sensitivity of $[\mathrm{Na} / \mathrm{H}]$ to a change of $50 \mathrm{~K}$ in $T_{\text {eff }}$ is only 0.04 dex (Carretta et al. 2007), and to bring the average $[\mathrm{Na} / \mathrm{H}] \sim$ -1.6 dex down to about -2.3 (to match the sequence of lowNa stars at the metallicity of NGC 6752) would require a change or error of $875 \mathrm{~K}$ in temperature, which is frankly unrealistic.

We could then safely proceed to the comparison between the distributions in $[\mathrm{Na} / \mathrm{H}]$ of field and cluster stars. An enlargement of Fig. 6 is shown in Fig. 8. From this figure it is evident that

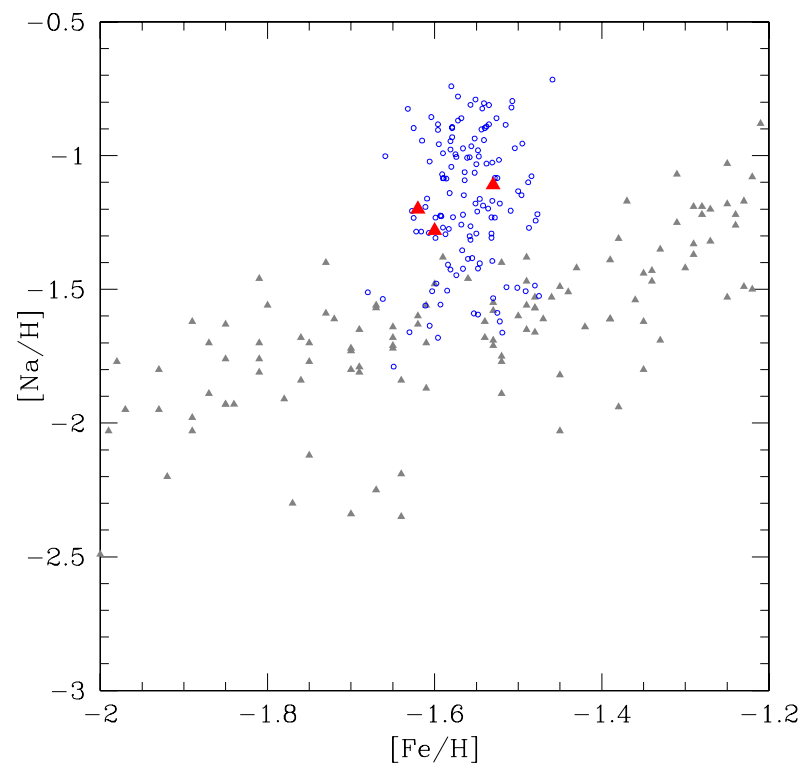

Fig. 9. $[\mathrm{Na} / \mathrm{H}]-[\mathrm{Fe} / \mathrm{H}]$ plane in the metallicity range centred on NGC 6752 (open blue symbols). Grey triangles are field stars and larger red triangles highlight three field stars with high $\mathrm{Na}$ abundance superimposed to stars in NGC 6752.

the division in components as defined in Carretta et al. (2009a) using both $\mathrm{Na}$ and $\mathrm{O}$ abundances is approximatively still valid.

A few outlier field stars can be seen superimposed on the stars in NGC 6752: HD 93529, BD+54 1323, and BD+52 1601 (Fig. 9). It is however dubious that thay could represent new cases of second-generation GC stars lost to the field. The first object is from the $\mathrm{H}$ sample, it is found on a retrograde orbit, and it could have been originated in a fragment accreted in the Galaxy, maybe experiencing a peculiar chemical evolution. Travaglio et al. (2004) reported BD+54 1323 among the stars suspected of AGB contamination, likely by mass transfer from a past companion, since for this star Burris et al. (2000) measured high values of light $s$-process elements. Finally, BD+52 1601 is listed in the Fourth Catalog of Interferometric Measurements of Binary Stars with a period of nine hours.

Excluding these three outliers, we estimated that the upper envelope of the field star distribution can be put at $[\mathrm{Na} / \mathrm{H}] \sim$ -1.3 dex (Fig. 10), by comparing the distribution of field and cluster stars in the metallicity range $-1.7<[\mathrm{Fe} / \mathrm{H}]<-1.4$ dex. This value corresponds closely to the $3-\sigma$ upper deviation $([\mathrm{Na} / \mathrm{H}]=-1.33 \mathrm{dex})$ from the field star relation obtained by eliminating iteratively all the outliers around the fit $[\mathrm{Na} / \mathrm{H}]$ vs. $[\mathrm{Fe} / \mathrm{H}]$ in the metallicity range $-2.4<[\mathrm{Fe} / \mathrm{H}]<-0.3 \mathrm{dex}$, as suggested by the referee.

Adopting the limit in $[\mathrm{Na} / \mathrm{H}]$ at -1.3 dex as the boundary between first- and second-generation stars in NGC 6752, we counted 36 and 97 stars in the two groups, respectively. Considering a total of 133 stars with $\mathrm{Na}$ abundances, we then derived fractions of $27 \pm 5 \%$ and $73 \pm 7 \%$ for the first and second generation components in NGC 6752, where the associated errors are from the Poisson statistics. These values are in perfect agreement with the fractions $27 \pm 5 \%(\mathrm{P})$ and $73 \pm 9 \%(\mathrm{I}+\mathrm{E}) \mathrm{ob}-$ tained in Carretta et al. (2009a) by using only the 98 stars with both $\mathrm{Na}$ and $\mathrm{O}$ abundances.

This new method is conceptually similar to the one employed in Carretta et al. (2009a), but the present variation allowed us to make use of all stars with a measured $\mathrm{Na}$ abundances, 


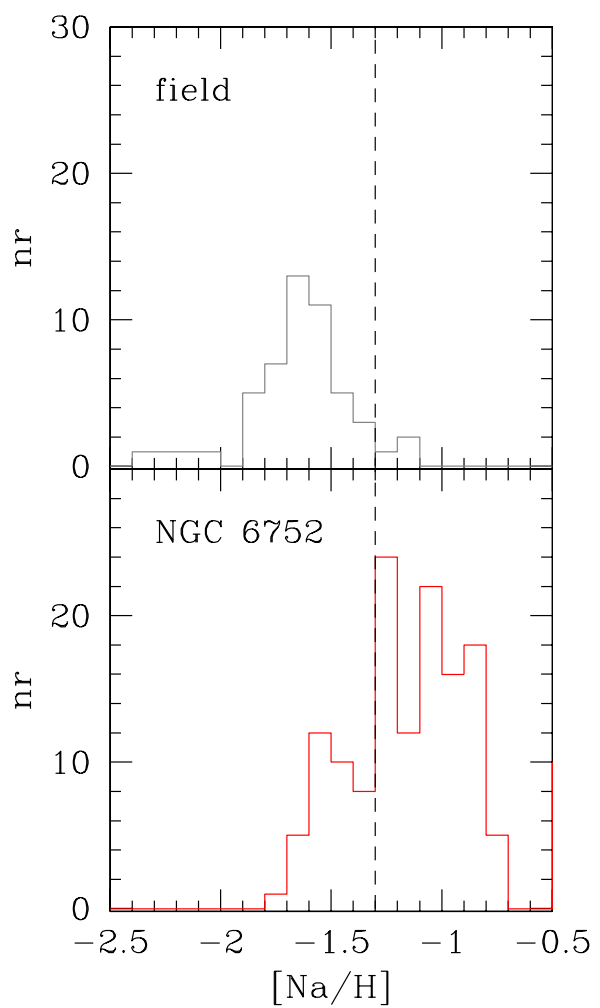

Fig. 10. Distribution of $[\mathrm{Na} / \mathrm{H}]$ ratios in the metallicity range $-1.7<$ $[\mathrm{Fe} / \mathrm{H}]<-1.4$ dex for field (upper panel) and cluster stars (lower panel). The dashed line fixes the upper envelope of the field stars distribution and the boundary between first- and second-generation stars in NGC 6752.

decreasing the statistical errors because it is usually easier to measure abundances of elements that are enhanced (and not depleted) in the proton-capture reactions and that have much stronger line strengths. Obviously, the present method is blind to the separation between the intermediate and extreme components of second-generation stars.

For a further consistency check we used also the smaller sample of 37 stars along the RGB in NGC 6752 presented in Yong et al. (2005). $\mathrm{Na}$ and $\mathrm{Fe}$ abundances were derived from spectra generally of higher resolution and $\mathrm{S} / \mathrm{N}$ than most stars in Carretta et al. (2007); these abundances were obtained using different scales of atmospheric parameters and line lists. However, there are 14 stars in common between the two samples: the differences in $[\mathrm{Fe} / \mathrm{H}]$ and $[\mathrm{Na} / \mathrm{H}]$ (in the sense Carretta minus Yong) are shown in Fig. 11.

On average, the offset in $[\mathrm{Fe} / \mathrm{H}]$ is not significant $(0.046 \mathrm{dex}$, with an rms scatter of $0.041 \mathrm{dex}, 14$ stars). However, a clear trend as a function of temperature does exist, probably owing almost entirely to the NLTE corrections for Na (adopted from Gratton et al. 1999 in Carretta et al., neglected in Yong et al.). They are relevant since both samples cover a large interval in luminosity and temperature along the RGB in this nearby cluster. We then fitted a linear regression through the data in the lower panel of Fig. 11 and derived the correction as a function of the effective temperatures by Yong et al. required to shift their abundances to the system used in the present work.

The separation between first and second generations at $[\mathrm{Na} / \mathrm{H}]=-1.3 \mathrm{dex}$, also in the case of the corrected data by Yong et al. (Fig. 12), gives values in good agreement with our previous findings. We found that 11 stars out of $37(30 \pm 9 \%)$ have a primordial composition consistent with that of field stars,

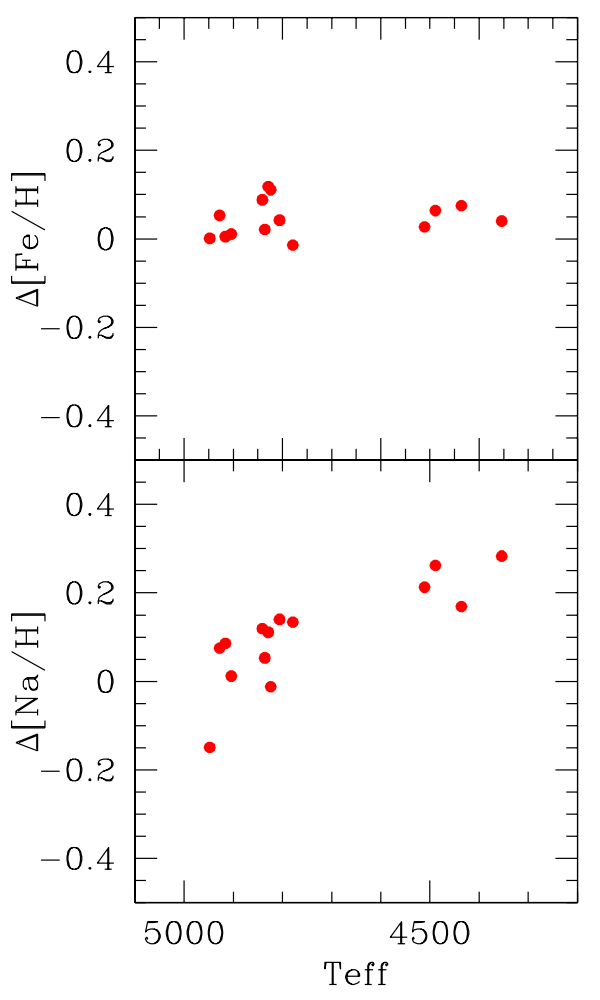

Fig. 11. Comparison of the differences in $[\mathrm{Na} / \mathrm{H}]$ (lower panel) and $[\mathrm{Fe} / \mathrm{H}]$ (upper panel), in the sense Carretta et al. (2007) minus Yong et al. (2005), as a function of the effective temperatures by Yong et al. (2005) for 14 stars analysed in both studies.

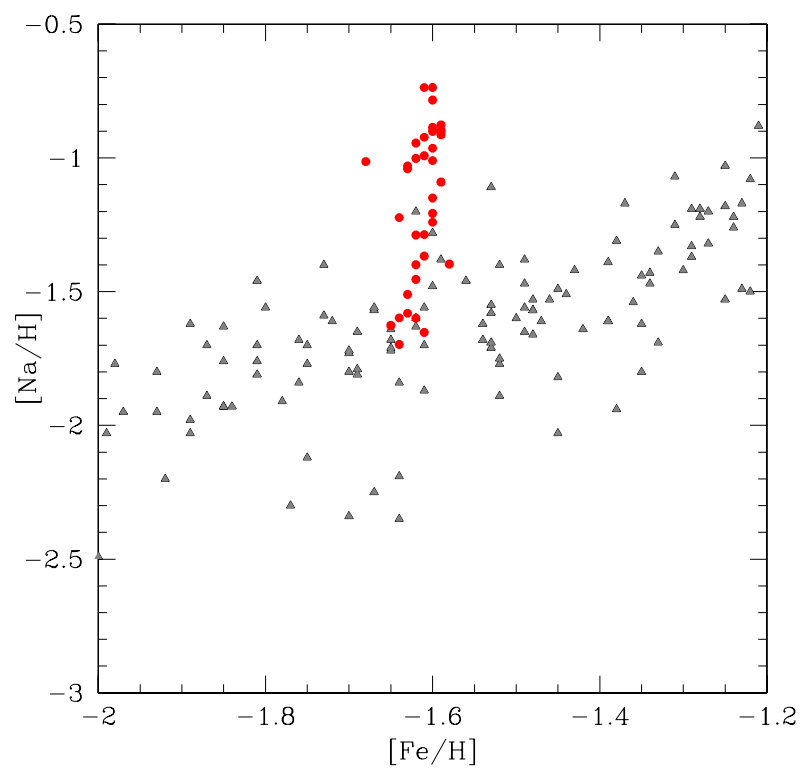

Fig. 12. As in Fig. 8, but using for NGC 6752 the 37 stars from Yong et al. (2005; red circles), corrected to the system of $\mathrm{Na}$ and $\mathrm{Fe}$ abundances in the present work.

whereas 26 stars $(70 \pm 14 \%$ of the sample) belong to the second generation in NGC 6752.

\section{Final thoughs on the formation of the Galactic halo}

In the present work we discussed how second-generation stars in GCs may be selected from their excess in $\mathrm{Na}$ above the 
level established in field stars. However, there are also a few field stars with chemical signatures that indicate a very likely origin in GCs. Some begin to be serendipitously individuated thanks to their $\mathrm{Na}$ excesses (Carretta et al. 2010; Ramírez et al. 2012); in other cases, focused studies specifically searched for second-generation GC stars lost to the field by looking at excess in the CN band strength (Martell et al. 2011).

Stars with the typical chemistry of second-generation stars in GCs are thus fairly easy to be recognised in the Galactic field. However, we have indirect evidence that the bulk of the contribution of GCs to the halo must be in form of stars bearing the primordial composition of first-generation stars. This conclusion stems (i) from the observed proportion of the stellar generations in GCs ( $\sim 30 \%$ primordial, $\sim 70 \%$ polluted); (ii) from the evidence that the second-generation stars are formed from gas polluted by a fraction only of first-generation stars (the most massive); and (iii) from consequent theoretical considerations and modelling (see e.g. Bekki et al. 2007; D'Ercole et al. 2008; Vesperini et al. 2010; Schaerer \& Charbonnel 2011, and references therein). All these studies invoke a massive loss of almost all the primordial stellar generation in early GCs (that were likely several times more massive than present-day GCs), to correctly reproduce the currently observed ratio of multiple populations without the need for ad hoc initial mass functions (IMFs). These stars would be not distinguished from normal halo field stars from their chemical composition alone (the foundation of the present approach), but, if present, they would be intermingled with the bulk of the field stellar distribution. However, we also signaled the presence of outliers, field stars with low $\mathrm{Na}$ abundances, that are claimed by several studies to come from small fragments accreted in the halo.

A not exhaustive comparison of this component with the chemical pattern of a few dwarf spheroidals (dSphs) is given in Fig. 13. Most of the giants studied in dSphs have a tendency to lie below the main distribution of Galactic field stars in the $[\mathrm{Na} / \mathrm{H}]-[\mathrm{Fe} / \mathrm{H}]$ plane (see also Fig. 12 in Tolstoy et al. 2009, with the more classical $[\mathrm{Na} / \mathrm{Fe}]$ ratios). These stars agree well with the accretion component of the Galaxy. Unfortunately, we have no stars in common with the analyses in dSphs to bring them on our common system. However, the same line of reasoning as above suggests that different studies should be consistent within a few hundreths of dex, or else the temperatures would be wrong by several hundred Kelvin, an unpalatable option.

The results of this triple comparison (Galactic field stars, GCs, dSphs) therefore seem to suggest that at least two classes of objects contributed to the building up of the Galactic halo. On one hand, some fragments were similar to the present-day dwarf spheroidal still orbiting our Galaxy, in particular in the low metallicity regime (see also Tolstoy et al. 2009). On the other hand, a larger fraction of the halo seems to have formed in larger fragments. Their higher mass allowed the same metallicity level of the component of the Galaxy undergoing dissipative collapse, at the same epoch, to be quickly reached. The ensuing location of first-generation stars lost at early epochs in GCs and of the field stars of the dissipative component is then indistinguishable, at present, which corroborates this dual channel for the origin of part of the halo.

Acknowledgements. This work was partially funded by the PRIN INAF 2011 grant "Multiple populations in globular clusters: their role in the Galaxy assembly" (PI E. Carretta). We thank Angela Bragaglia for valuable suggestions and discussions. We also wish to thank the referee for useful comments. This research has made extensive use of NASA's Astrophysical Data System and the SIMBAD database (in particular Vizier), operated at the CDS, Strasbourg, France, without which this work could not have been done. We think that these databases are fundamental tools for advancing in Astrophysics, and we wish to

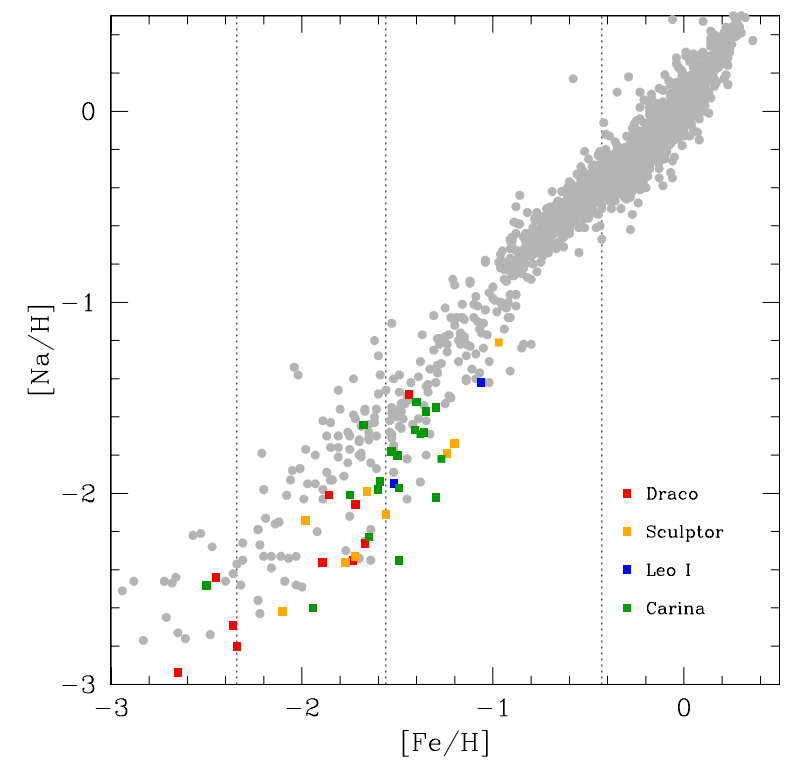

Fig. 13. Comparison of our final sample of field stars with stars in four dwarf spheroidals: Draco (Cohen \& Huang 2009; Shetrone et al. 2001), Sculptor (Kirby \& Cohen 2012; Shetrone et al. 2003; Geisler et al. 2005), Carina (Shetrone et al. 2003; Venn et al. 2012; Koch et al. 2008), and Leo I (Shetrone et al. 2003).

warmly thank all colleagues who in the true scientific spirit contribute their data to these facilities.

\section{References}

Adibekyan, V. Zh., sousa, S. G., Santos, N. C., et al. 2012, A\&A, 545, A32 (A) Andrievsky, S. M., Spite, M., Korotin, S. A., et al. 2007, A\&A, 464, 1081 Baumüller, D., Butler, K., \& Gehren, T. 1998, A\&A, 338, 637

Bekki, K., Campbell, S. W., Lattanzio, J. C., \& Norris, J. E. 2007, MNRAS, 377, 335

Bensby, T., Feltzing, S., Lundström, I., \& Ilyin, I. 2005, A\&A, 433, 185 (B)

Burris, D. L., Pilachowski, C. A., Armandroff, T. E., et al. 2000, ApJ, 544, 302 Calamida, A., Bono, G., Stetson, P. B., et al. 2007, ApJ, 670, 400

Carollo, D., Martell, S. L., Beers, T. C., \& Freeman, K. C. 2013, ApJ, 769, 87

Carretta, E., Gratton, R. G., \& Sneden, C. 2000, A\&A, 356, 238 (CA)

Carretta, E., Gratton R. G., Lucatello, S., Bragaglia, A., \& Bonifacio, P. 2005, A\&A, 433, 597

Carretta, E., Bragaglia, A., Gratton R. G., et al. 2006, A\&A, 450, 523

Carretta, E., Bragaglia, A., Gratton, R. G., Lucatello, S., \& Momany, Y. 2007, A\&A, 464, 927

Carretta, E., Bragaglia, A., Gratton, R. G., et al. 2009a, A\&A, 505, 117

Carretta, E., Bragaglia, A., Gratton, R. G., \& Lucatello, S. 2009b, A\&A, 505, 139

Carretta, E., Bragaglia, A., Gratton, R. G., D’Orazi, V., \& Lucatello, S. 2009c, A\&A, 508, 695

Carretta, E., Bragaglia, A., Gratton, R. G., et al. 2010, A\&A, 519, A60

Carretta, E., Bragaglia, A., Gratton, R. G., D’Orazi, V., \& Lucatello, S. 2011, A\&A, 535, A121

Carretta, E., Bragaglia, A., Gratton, R. G., Lucatello, S., \& D’Orazi, V. 2012, ApJ, 750, L14

Cassisi, S., Mucciarelli, A., Pietrinferni, A., Salaris, M., \& Ferguson, J. 2013, A\&A, 554, A19

Chen, Y. Q., Nissen, P. E., Zhao, G., Zhang, H. W., \& Benoni, T. 2000, A\&AS, 141, 491 (C)

Cohen, J. G. 1978, ApJ, 223, 487

Cohen, J. G., \& Huang, W. 2009, ApJ, 701, 1053

Denisenkov, P. A., \& Denisenkova, S. N. 1989, A. Tsir., 1538, 11

D’Ercole, A., Vesperini, E., D’Antona, F., McMillan, S. L. W., \& Recchi, S. 2008, MNRAS, 391, 825

Edvardsson, B., Andersen, J., Gustafsson, B., et al. 1993, A\&A, 275, 101

Eggen, O. J., Lynden-Bell, D., \& Sandage, A. R. 1962, ApJ, 136, 748

Fulbright, J. P. 2000, AJ, 120, 1841 (F)

Fulbright, J. P. 2002, AJ, 123, 404

Gehren, T., Liang, Y. C., Shi, J. R., Zhang, H. W., \& Zhao, G. 2004, A\&A, 413, 1045 
Gehren, T., Shi, J. R., Zhang, H. W., Zhao, G., \& Korn, A. J. 2006, A\&A, 451, 1065 (GE)

Geisler, D., Smith, V. V., Wallerstein, G., Gonzalez, G., \& Charbonnel, C. 2005, AJ, 129,1428

Gratton, R. G., Carretta, E., Eriksson, K., \& Gustafsson, B. 1999, A\&A, 350, 955

Gratton, R. G., Sneden, C., Carretta, E., \& Bragaglia, A. 2000, A\&A, 354, 169 (GR)

Gratton, R. G., Bonifacio, P., Bragaglia, A., et al. 2001, A\&A, 369, 87

Gratton, R. G., Carretta, E., Claudi, R., Lucatello, S., \& Barbieri, M. 2003, A\&A, 404, 187 (G)

Gratton, R. G., Sneden, C., \& Carretta, E. 2004, ARA\&A, 42, 385

Gratton, R. G., Carretta, E., \& Bragaglia, A. 2012, A\&ARv, 20, 50

Grundahl, F., Briley, M., Nissen, P. E., \& Feltzing, S. 2002, A\&A, 385, L14

Hanson, R. B., Sneden, C., Kraft, R. P., \& Fulbright, J. 1998, AJ, 116, 1286 (H)

Ivans, I. I., Sneden, C., James, C. R., et al. 2003, ApJ, 592, 906

Johnson, J. A. 2002, ApJS, 139, 219

Jonsell, K., Edvardsson, B., Gustafsson, B., et al. 2005, A\&A, 440, 321 (J)

King, J. R. 1997, AJ, 113, 2302

Kirby, E. N., \& Cohen, J. G. 2012, AJ, 144, 168

Koch, A., Grebel, E. K., Gilmore, G. F., et al. 2008, AJ, 135, 1580

Korotin, S. A., \& Mishenina, T. V. 1999, A. Rep., 43, 533

Kraft, R. P. 1994, PASP, 106, 553

Langer, G. E., Hoffman, R., \& Sneden, C. 1993, PASP, 105, 301

Lind, K., Asplund, M., Barklem, P. S., \& Belyaev, A. K. 2011, A\&A, 528, A103

Martell, S. L., Smolinski, J. P., Beers, T. C., \& Grebel, E. K. 2011, A\&A, 534, A136

Mashonkina, L. I., Shimanskii, V. V., \& Sakhibullin, N. A. 2000, A. Rep., 44, 790

Milone, A., Piotto, G., Bedin, L., et al. 2012, ApJ, 744, 58

Milone, A. P., Marino, A. F., Piotto, G., et al. 2013, ApJ, 767, 120

Mishenina, T. V., Kovtyukh, V. V., Korotin, S. A., \& Soubiran, C. 2003, A. Rep., 47, 422 (M3)

Mishenina, T. V., Gorbaneva, T. I., Basak, N. Yu., Soubiran, C., \& Kovtyukh,

V. V. 2011, A. Rep., 55, 689 (M1)

Nissen, P. E., \& Schuster, W. J. 1997, A\&A, 326, 751
Nissen, P. E., \& Schuster, W. J. 2010, A\&A, 511, L10 (N)

Nissen, P. E., \& Schuster, W. J. 2011, A\&A, 530, A15

Peterson, R. C. 1980, ApJ, 237, L87

Pilachowski, C. A., Sneden, C., \& Kraft, R. P. 1996, AJ, 111, 1689

Prochaska, J., Naumov, S. O., Carney, B. W., McWilliam, A., \& Wolfe, A. M. 2000, AJ, 120, 2513

Ramírez, I., Meléndez, J., \& Chanamé, J. 2012, ApJ, 757, 164 (R12)

Reddy, B. E., Lambert, D. L., \& Allende Prieto, C. 2006, MNRAS, 367, 1329 (R)

Renzini, A., \& Fusi Pecci, F. 1988, ARA\&A, 26, 199

Sbordone, L., Salaris, M., Weiss, A., \& Cassisi, S. 2011, A\&A, 534, A9

Schaerer, D., \& Charbonnel, C. 2011, MNRAS, 413, 2297

Schuster, W. J., Moreno, E., Nissen, P. E., \& Pichardo, B. 2012, A\&A, 538, A21

Searle, L., \& Zinn, R. 1978, ApJ, 225, 357

Shetrone, M. D., Côté, P., \& Sargent, W. L. W. 2001, ApJ, 548, 592

Shetrone, M., Venn, K. A., Tolstoy, E., et al. 2003, AJ, 125, 684

Smith, G. H. 1987, PASP, 99, 67

Smith, G. H., \& Martell, S. L. 2003, PASP, 115, 1211

Soubiran, C., \& Girard, P. 2005, A\&A, 438, 139

Stephens, A., \& Boesgaard, A. M. 2002, AJ, 123, 1647

Takeda, Y., Zhao, G., Takada-Hidai, M., et al. 2003, Chin. J. Astron. Astrophys., 3,316

Tolstoy, E., Hill, V., \& Tosi, M. 2009, ARA\&A, 47, 371

Travaglio, C., Gallino, R., Arnone, E., et al. 2004, ApJ, 601, 864

Truran, J. W., \& Arnett, W. D. 1971, A\&AS, 11, 430

Venn, K. A., Irwin, M., Shetrone, M. D., et al. 2004, AJ, 128, 1177

Venn, K. A., Shetrone, M. D., Irwin, M. J., et al. 2012, ApJ, 751, 102

Vesperini, E., McMillan, S. L. W., D’Antona, F., \& D’Ercole, A. 2010, ApJ, 718, 112

Vesperini, E., McMillan, S. L. W., D’Antona, F., \& D’Ercole, A. 2013, MNRAS, 429,1913

Woosley, S. E., \& Weaver, T. A. 1995, ApJS, 101, 181

Yong, D., Grundahl, F., Lambert, D. L., Nissen, P. E., \& Shetrone, M. D. 2003, A\&A, 402, 985

Yong, D., Grundahl, F., Nissen, P. E., Jensen, H. R., \& Lambert, D. L. 2005, A\&A, 438, 875

Yong, D., Grundahl, F., Johnson, J. A., \& Asplund, M. 2008, ApJ, 684, 1159

Zolotov, A., Willman, B., Brooks, A. M., et al. 2009, ApJ, 702, 1058 